\title{
FIXED POINTS AND COMPACT WEIGHTED COMPOSITION OPERATORS ON BANACH WEIGHTED HARDY SPACES
}

\author{
ZAHRA FATTAHI VANANI*, ALI ILOON KASHKOOLY** AND BAHMANN YOUSEFI*** \\ *Department of Mathematics, College of Sciences \\ Yasouj University, Yasouj, 75914-74831, Iran \\ E-mail: zfattahi@mail.yu.ac.ir \\ ** Department of Mathematics, College of Sciences \\ Yasouj University, Yasouj, 75914-74831, Iran \\ E-mail: kashkooly@mail.yu.ac.ir \\ *** Department of Mathematics, Payame Noor University \\ P.O. Box 19395-3697, Tehran, Iran \\ E-mail: b_yousefi@pnu.ac.ir
}

\begin{abstract}
We consider weighted composition operators $C_{\psi, \varphi}$ acting on Banach weighted Hardy spaces in the open unit disk such that the norms of the kernel functions for the appropriate order derivatives tend to infinity as one approaches the boundary. We investigate the relation between the compactness of $C_{\psi, \varphi}$, the angular derivatives and the fixed points of $\varphi$, and we will see that compactness of $C_{\psi, \varphi}$ for some weight functions $\psi$ forces $\varphi$ to have a fixed point inside the open unit disk.

Key Words and Phrases: Banach weighted Hardy spaces, bounded point evaluation, weighted composition operator, Denjoy-Wolff point, fixed point.

2010 Mathematics Subject Classification: 47B33, 47B37.
\end{abstract}

Acknowledgments. The authors are grateful to the referees for careful reading of the manuscript. Their remarks motivated the authors to make some valuable improvements.

\section{REFERENCES}

[1] L. Ahlfors, Conformal Invariants, McGraw-Hill, New York, 1973.

[2] P.S. Bourdon, S.K. Narayan, Normal weighted composition operators on the Hardy space, J. Math. Anal. Appl., 367(2010), no. 1, 278-286.

[3] J.G. Caughran, H.J. Schwartz, Spectra of compact composition operators, Proc. Amer. Math. Soc., 51(1975), no. 1, 127-130.

[4] D.D. Clahane, Spectra of compact composition operators over bounded symmetric domains, Integral Equations and Operator Th., 51(2005), no. 1, 41-56.

\footnotetext{
${ }^{* *}$ Correspondence author.
} 
[5] D.D. Clahane, Compact weighted composition operators and fixed points in convex domains, Fixed Point Theory Appl., Vol. 2007, ID 28750, 8 pages.

[6] F. Colonna, S. Li, Weighted composition operators from the Bloch space and the analytic Besov spaces into the Zygmund space, J. Operators, Vol. 2013, Article ID 154029, 9 pages.

[7] M.D. Contreras, A.G. Hernandez-Dias, Weighted composition operators in weighted Banach spaces of analytic functions, J. Austral. Math. Soc. Series A, 69(2000), 41-60.

[8] M.D. Contreras, A.G. Hernandez-Dias, Weighted composition operators on weighted Hardy spaces, J. Math. Anal. Appl., 263(2001), 224-233.

[9] C.C. Cowen, B.D. MacCluer, Composition Operators on Spaces of Analytic Functions, Studies in Advanced Mathematics, CRC Press, Boka Raton, Fla, USA, 1995.

[10] C.C. Cowen, G. Gunatillake, E. Ko, Hermitian weighted composition operators and Bergman extremal functions, Complex Anal. Operator Th., 7(2013), no. 1, 69-99.

[11] M. Fatehi, M.H. Shaabani, Some essentially normal weighted composition operators $n$ the weighted Bergman spaces, Complex Var. Elliptic Equ., 60(2015), 1205-1216.

[12] G. Gunatillake, Spectrum of a compact weighted composition operator, Proc. Amer. Math. Soc., 135(2007), no. 2, 461-467.

[13] G. Gunatillake, Compact weighted composition operators on the Hardy space, Proc. Amer. Math. Soc., 136(2008), no. 8, 2895-2899.

[14] G. Gunatillake, Invertible weighted composition operators, J. Funct. Anal., 261(2011), no. 3, $831-860$.

[15] G. Gunatillake, M. Jovovich, W. Smith, Numerical ranges of weighted composition operators, J. Math. Anal. Appl., 413(2014), no. 1, 458-475.

[16] Z. Kamali, B. Yousefi, Disjoint hypercyclicity of weighted composition operators, Proc. Ind. Acad. Sci. (Math. Sci.), 125(2015), no. 4, 559-567.

[17] V. Matache, Weighted composition operators on $H^{2}$ and applications, Complex Anal. Operator Th., 2(2008), 169-197.

[18] J. Shapiro, On Composition Operators and Classical Function Theory, Springer-Verlag, 1993.

[19] A.M. Sinclair, Automatic Continuity of Linear Operators, London Math. Soc., Lecture Notes Ser. 21, Cambridge Univ. Press, 1976.

[20] S. Ye, G. Hu, Weighted composition operators on the Zygmund space, Abstract and Applied Anal., Volume 2012, Article ID 462482, 18 pages.

[21] B. Yousefi, Unicellularity of the multiplication operator on Banach spaces of formal power series, Studia Mathematica, 147(2001), no. 3, 201-209.

[22] B. Yousefi, Bounded analytic structure of the Banach space of formal power series, Rendiconti Del Circolo Matematico Di Palermo Serie II, LI (2002), 403-410.

[23] B. Yousefi, Strictly cyclic algebra of operators acting on Banach spaces $H^{p}(\beta)$, Czechoslovak Math. J, 54(2004), no. 129, 261-266.

24] B. Yousefi, A.I. Kashkuli, Cyclicity and unicellularity of the differentiation operator on Banach spaces of formal power series, Mathematical Proc. of the Royal Irish Academy, 105A(2005), no. 1, 1-7.

[25] B. Yousefi, On the eighteenth question of Allen Shields, International J. Math., 16(2005), no. $1,37-42$.

[26] B. Yousefi, H. Rezaei, Hypercyclic property of weighted composition operators, Proc. Amer. Math. Soc., 135(2007), no. 10, 3263-3271.

[27] B. Yousefi, J. Izadi, Weighted composition operators and supercyclicity criterion, International J. Math Mathematical Sciences, Vol. 2011, Article ID 514370, 5 pages.

[28] Z.H. Zhou, C. Yuan, Spectrum of compact weighted composition operators on the weighted Hardy spaces in the unit ball, J. Ineq. Appl., 2007(2007), ID 61313, 8 pages.

Received: October 12, 2015; Accepted: January 19, $201 \%$. 
